\title{
Buckling behavior of carbon nanotubes under bending: from ripple to kink
}

\author{
C. G. Wang ${ }^{1,4 *}$, Y. P. Liu ${ }^{1}$, J. Al-Ghalith ${ }^{2}$, T. Dumitrică ${ }^{2}$, M. K. Wadee ${ }^{3}$, H .F. Tan ${ }^{1,4}$
}

${ }^{1}$ Center for Composite Materials, Harbin Institute of Technology, Harbin, 150001, China

${ }^{2}$ Department of Mechanical Engineering, University of Minnesota, Minneapolis, Minnesota 55455, USA

${ }^{3}$ College of Engineering, Mathematics and Physical Sciences, University of Exeter, North Park Road, Exeter, Devon EX4 4QF, United Kingdom.

${ }^{4}$ National Key Laboratory of Science and Technology on Advanced Composites in Special Environments, Harbin Institute of Technology, Harbin 150080, China.

\begin{abstract}
This paper elucidates the buckling behavior of carbon nanotubes (CNTs) under bending. CNTs are modeled as continuous thin-wall circular tubes, and their buckling is governed by equations that take into account of the sectional Brazier effect and non-uniform structural deformation. The CNT governing equations (fourth-order ordinary differential nonlinear equations with integral conditions) are solved by introducing a continuation algorithm. In addition, the buckling behavior of CNTs under bending is simulated with objective molecular dynamics (OMD). The atomistic simulations are used to verify the continuum results. We show that there exist lowand high-strain phases during the bending process of CNTs, and the transition in between may divide the whole bending process into three stages: low-curvature stage, mixed-curvature stage and high-curvature stage. Ripples are generated on the CNT surfaces before the formation of kinks. Compared to single-walled CNTs (SWCNTs), hydrogen-filled CNTs have a longer mixed-strain stage owing to the presence of internal pressure, and are therefore more inclined to exhibit a ripple morphology. Our results offer better understanding of the buckling behavior of CNTs, and may open up new opportunities for the design and applications of novel CNT-based nanoelectronics.
\end{abstract}

${ }^{*}$ Corresponding author E-email: wangcg@ hit.edu.cn 
Keywords: Carbon nanotubes; Buckling behaviour; Phase transformation; ripples and kinks

\section{Introduction}

Carbon nanotubes (CNTs), as a new allotrope of the carbon family, have a unique one-dimensional (1D) tubular architecture that is composed of single- or multi-layer graphene nanosheets rolled up in a seamless way [1]. The extraordinary electrical, optical, mechanical and thermal properties of CNTs are intimately related to their deformation [2], [3]. Buckling, as a typical instability mode and complex nonlinear deformation of CNTs [4], [5], may dramatically alter their inherent structural equilibrium and thus lead to a series of changes in properties [6]. As such, CNTs in the post-buckling state have potential uses as excellent single-electron transistors [7], or gas and water pipelines [8]. In this context, the buckling behavior of CNTs under different loads has provoked a surge of research interest recently [9], [10], [11]. For example, previous studies have shown that a number of possible morphologies may occur in CNTs under bending, including increase of curvature, sectional Brazier effect, ripples, kinks and Yoshimura patterns [12], [13], [14]. How comprehensively to evaluate these possible morphologies, as well as their relationship and formation mechanism, remains a challenge [15].

Thus far, several experimental approaches have been proposed qualitatively to observe the buckling behavior of CNTs [16], [17]. However, quantitative analysis of CNT buckling remains a tough task owing to the lack of accurate characterization techniques. The formation and evolution mechanics of CNT buckling instabilities is still not yet well understood [18]. Continuum theory and molecular dynamics simulation strategies have been proposed in response, and have become effective means for exploring the buckling behavior of CNTs. In the continuum theory approach, CNTs are generally represented as the elastic beams [19], [20], elastic shells [21], [22], or with nonlocal theory [23], [24], [25] and space frame models [26], [27].

The investigations of thin elastic shell tubes date back to the 1920s when Brazier [28] demonstrated that the initial bending behaviour of tubes is characterized by a uniform ovalization of the cross-section. Then Reissner [29] generalized the theory 
and derived series expansions which successfully modelled the observed moment-curvature relationship, as a curve with monotonically decreasing slope. Axelrad [30] presented a nonlinear formulation for the ovalization instability of tubular members under bending based on a nonlinear flexible shell theory. Results were reported for initially straight and bent tubes, including the effects of pressure. However, the transition from uniform ovalization to localized deformation in the form of kinking has proved difficult to model mathematically. Karamanos and Tassoulas [31] solved this problem by using nine-node degenerated shell elements, and kinematic constraints to enforce uniform ovalization along the tube. In subsequent works, a similar 'tube element' model is proposed [32] [33], which considered the ovalized pre-buckling state and the effect of pressure. A special non-linear finite element method was used to determine the bifurcation point on the ovalization path of isotropic elastic shell and its rippling configuration.

Most continuum studies have concentrated on the buckling morphologies of CNTs, as well as on the relationship between the rippling wavelength or amplitude and tube curvature [18]. On the other hand, molecular dynamics simulations have been widely employed for the analysis of CNT buckling as an effective means of conducting numerical experiments [34], [35], [36]. It is worth noting, however, that the traditional molecular dynamics approach has several inherent disadvantages for the simulation of CNT buckling instability. Firstly, bending does not obey translational symmetry assumed by traditional molecular dynamics, and the periodic boundary conditions (PBC) are thus invalid in this case. Second, traditional molecular dynamics has to fix the atoms on both ends of CNTs to apply a bending moment, which has the disadvantage of introducing spurious end and fixed boundary effects [13].

In this paper we provide a comprehensive study of the buckling instability mode in CNTs from a continuum mechanics perspective. The study is structured as follows. In section 2, the CNT buckling governing equations, taking account of the sectional Brazier effect, are established. The aim here is to describe different morphologies of CNTs under bending, including smooth bending, ripples and kinks. In section 3, the governing equations (fourth-order ordinary differential nonlinear equations with integral conditions) are solved by introducing a continuation algorithm to acquire the equilibrium paths and buckling characteristics of CNTs under bending. In section 4, 
the phase transitions of CNTs during the bending process are elucidated by using the non-convex energy theory, thereby revealing their buckling mechanisms. Then, the buckling behavior of CNTs under bending is undertaken by simulation analysis and verified with objective molecular dynamics (OMD). Finally, the effects on structural performance and buckling of the CNT by filling its internal cavity with hydrogen molecules are discussed.

\section{Model formulation}

\subsection{Continuum modeling}

In terms of structural mechanics, a single-walled carbon nanotube (SWCNT) can be viewed as a typical thin-wall structure. As such, a SWCNT is equivalent to a continuous thin-walled circular tube and can be studied using conventional continuum mechanics. In our study we investigate the behavior of a straight SWNT with a radius of $R$ and a length of $L$. The structural parameters are described by a cartesian coordinate system, where the longitudinal direction is determined as the z-axis, the sectional horizontal direction as the $\mathrm{x}$-axis, and the sectional vertical direction as the y-axis (Fig. 1(a)). A steady moment $M$ is applied on each end of the nanotube about the $\mathrm{x}$-axis.

(a)

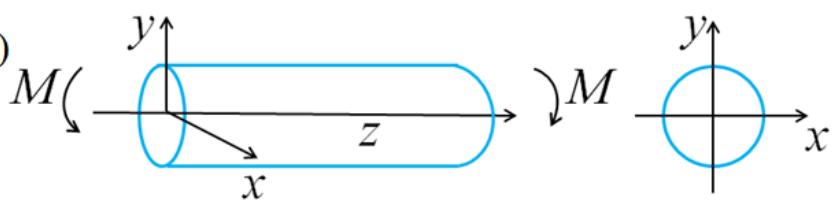

(b)

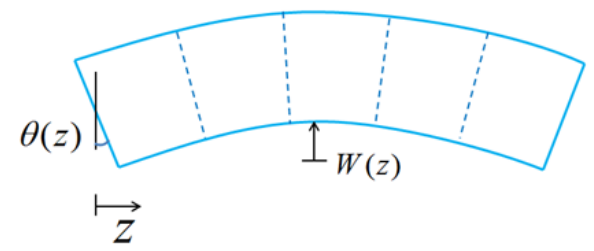

Fig. 1. Illustration of a SWCNT under bending: (a) orientation and coordinate axes of an undeformed nanotube, and (b) sway and tilt components.

Euler beam theory is adequate for the buckling behavior of a SWNT with a high aspect ratio $(L / R)$. However, this model overestimates the buckling load because that model neglects transverse shear deformations. Therefore, a more refined beam model, that allows for such deformations, namely Timoshenko beam theory, is employed to provide a more accurate description of the buckling behavior of a SWCNT. In Timoshenko's theory, we introduce two dimensionless factors representing the global 
sway and tilt, i.e., $q_{s}$ and $q_{t}$, directly to describe this shear effect, as shown in Fig. 1(b). Here $q_{s}$ need not equal to $q_{t}$, which is different from the Euler model where the two are assumed to be identical. Then, the global sway $W(z)$ and tilt $\theta(z)$ of the SWCNT can be approximated as the following polynomials [37]:

$$
\begin{aligned}
& W(z)=\frac{q_{s} z(z-L)}{L} \\
& \theta(z)=\frac{q_{t}(2 z-L)}{L}
\end{aligned}
$$

Furthermore, $\alpha$ is the dimensionless form of curvature $K$, and can be expressed as:

$$
\alpha=2 q_{t} \sqrt{\Delta}=\frac{2 q_{t} R^{2}}{L t} \sqrt{12\left(1-v^{2}\right)}
$$

where $\Delta=\frac{R^{4}}{12\left(1-v^{2}\right) L^{2} t^{2}}, v$ is the Poisson ratio, and $t$ is the wall thickness of the model tube.

Numerous previous reports have shown that CNT bending may result in lattice mismatch, and an obvious sectional Brazier effect may occur whose solution is beyond the reach of the Timoshenko beam model. Therefore, we seek to combine Timoshenko's theory with Reissner's theory [29], aiming to take into account of the sectional ovalization deformation during the bending process of the SWCNT. In the latter we assume point $A(x, y)$ on the initial circular section (with $\mathrm{O}$ as the central point). When the cross section is subjected to ovalization deformation, point $\mathrm{A}$ will shift to point $A^{\prime}(x+\varsigma, y+\eta)$, and $\varphi$ is the included angle between OA and the negative direction of y-axis, as shown in Fig. 2.

The sectional ovalization deformation is irrelevant to the length direction (z-axis), that is, Reissner's theory assumes that the sectional ovalization deformation is uniform along the length direction and cannot account for any nonlinear effects out of the cross-sectional plane, such as compressive buckling of the inner side. Actually, ripples or kinks may occur on the SWCNT surface when there is axial instability. Such instability may induce non-uniform sectional deformation along the longitudinal axis, which Reissner's theory does not account for. As such, an additional local radial displacement function $w(z, \varphi)$, associated with the length $(\mathrm{z})$ and the circumferential 
angle $(\varphi)$, is introduced to take into account of non-uniform sectional deformation. The concrete form of $w(z, \varphi)$ will be discussed in detail in Section 2.3. When $w(z, \varphi)$ is introduced, the coordinates of point $A^{\prime}$ will be updated to $A^{\prime \prime}$ $(x+\varsigma+w \sin \varphi, y+\eta-w \cos \varphi)$. Therefore, the introduction of $w(z, \varphi)$ can effectively describe non-uniform sectional deformation along the longitudinal direction under axial instability.

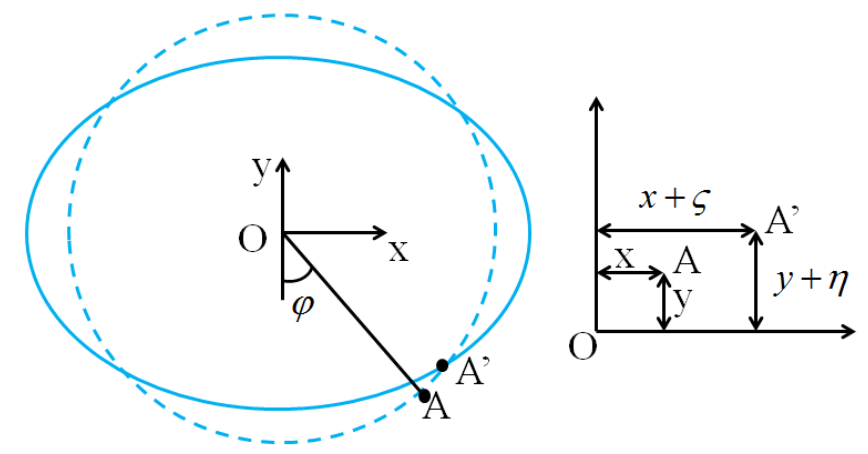

Fig. 2. Definitions of coordinates and deformation of a section of the carbon nanotube.

A SWCNT whose interior is adsorbed with substances has an effect on the wall of the tube which is equivalent to the application of internal pressure in continuum mechanics. The effect of this equivalent pressure should be determined by a combination of macroscopic continuum mechanics and microscopic molecular mechanics.

It is known that a CNT may undergo radial expansive deformation under internal pressure. According to the expansive deformation theory proposed by Le van and Wielgosz [38], the relationship between equivalent pressure $P$ and the radii after expansive deformation $R_{\phi}$ can be expressed as following (established in Appendix A):

$$
P=\frac{4 \sqrt{3}}{3(2-v)} \frac{\left(R_{\phi}-R\right)}{R^{2}}\left(\frac{1}{\left(\partial^{2} V / \partial r_{i j}^{2}\right)_{0}}+\frac{8}{B}\right)^{-1}
$$

\subsection{Buckling governing equations}

When nonlinear large deformation is taken into account, the axial total strain can be expressed as follows according to the von Karman hypothesis:

$$
\varepsilon=\varepsilon_{z}+\dot{u}_{\varphi}+\frac{1}{2} \dot{w}^{2}
$$


where $\varepsilon_{z}=\frac{y+\eta}{R}=\frac{2 q_{t}(y+\eta)}{L}$ is the axial strain term corresponding to the global bending of the CNT, $\dot{u}_{\varphi}+\frac{1}{2} \dot{w}^{2}$ is the axial strain term corresponding to the local deformation of the CNT and the notation "dot" above the variables denotes a spatial derivative $\mathrm{d} / \mathrm{d} z$.

Here we try to simplify the expression form of the total potential, that is, to reduce the original dual integrations to a single integration, which is advantageous for the simplification of the following buckling governing equations (partial differential equations) to ordinary differential equations. We assume that the in-plane deformation (u) is proportional to the distance to the $\mathrm{x}$-axis, that is,

$$
u(z, \varphi)=u(z) y / R=-u(z) \cos \varphi
$$

It also should be stressed that the symmetry of $w(z, \varphi)$ along y-axis should be guaranteed. Therefore, $w(z, \varphi)$ can be expressed as a Fourier series:

$$
w(z, \varphi)=\sum_{n=0}^{\infty} w_{i}(z) \cos i \varphi
$$

It is known that the higher number $n$ renders the results more accurate, while it makes the calculation more complex and difficult. Here we select $i=0,1$ and 2 corresponding to $w_{0}, w_{1}$ and $w_{2}$ deformation, where $w_{0}$ describes the axisymmetric deformation mode, $w_{1}$ is related to a mode with symmetry only about the y-axis, and $w_{2}$ is related to a nonaxisymmetric mode which maintains symmetry about the $\mathrm{x}$ - and $\mathrm{y}$-axes. The three parameters are adequate for reflecting the mode and characteristics of sectional local deformation. As such, the selected $i=0,1$ and 2 can satisfy the calculation accuracy as well as simplify the calculation. Therefore, the total potential, $\Pi$, can be expressed as (established in Appendix B): 


$$
\begin{aligned}
& \Pi=E t \pi R \int_{\phi}^{L}\left\{R _ { \phi } ^ { 2 } \left[\frac{q_{s}^{2}}{L^{2}}\left(2-\Delta q_{t}^{2}\right)+\left(\frac{1}{2}-\frac{\Delta}{4} q_{t}^{2}\right) \ddot{w}_{0}^{2}+\left(\frac{3}{8}-\frac{5 \Delta}{24} q_{t}^{2}\right) \ddot{w}_{1}^{2}+\left(\frac{1}{2}-\frac{\Delta}{3} q_{t}^{2}\right) \ddot{w}_{0} \ddot{w}_{2}\right.\right. \\
& \left.+\left(\frac{1}{4}-\frac{7 \Delta}{48} q_{t}^{2}\right) \ddot{w}_{2}^{2}\right]+\left[q_{t}^{2} \frac{R_{\phi}^{2}}{L^{2}}\left(2-\Delta q_{t}^{2}\right)-q_{t} \frac{R_{\phi}}{L} \dot{w}_{1} \dot{w}_{0}\left(2-\frac{\Delta}{2} q_{t}^{2}\right)-q_{t} \frac{R_{\phi}}{L} \dot{w}_{1} \dot{w}_{2}\left(1-\frac{\Delta}{3} q_{t}^{2}\right)\right. \\
& \left.+\frac{1}{2} \dot{u}^{2}+\frac{1}{4} \dot{w}_{0}^{4}+\frac{3}{4} \dot{w}_{0} \dot{w}_{1}^{2} \dot{w}_{2}+\frac{3}{4} \dot{w}_{0}^{2}\left(\dot{w}_{1}^{2}+\dot{w}_{2}^{2}\right)+\frac{1}{2} \dot{w}_{1}\left(2 \dot{w}_{0}+\dot{w}_{2}\right)+\frac{3}{32}\left(\dot{w}_{1}^{4}+4 \dot{w}_{1}^{2} \dot{w}_{2}^{2}+\dot{w}_{2}^{4}\right)\right] \\
& +\left(\frac{G}{2 E}+\frac{P R_{\phi}}{2 E t}\left(1-\frac{1}{9} \Delta^{2} q_{t}^{4}\right)\right)\left[2\left(q_{s}-q_{t}\right)^{2}+2 \frac{u^{2}}{R_{\phi}^{2}}+2 \dot{w}_{0}+\dot{w}_{1}+\dot{w}_{2}+\frac{8 z^{2}}{L^{2}}\left(q_{s}-q_{t}\right)^{2}\right. \\
& \left.+\frac{8 z \dot{w}_{0}}{L}\left(q_{s}-q_{t}\right)+4 \frac{u}{R_{\phi}}\left(\dot{w}_{0}+\frac{2 z}{L}\left(q_{s}-q_{t}\right)\right)-4\left(q_{s}-q_{t}\right)\left(\frac{u}{R_{\phi}}+\dot{w}_{0}+\frac{2 z}{L}\left(q_{s}-q_{t}\right)\right)\right] \\
& \left.+\frac{D}{2 E t R_{\phi}^{4}}\left(\dot{w}_{1}^{2}+16 \dot{w}_{2}^{2}\right)-\left(\frac{M}{\pi R_{\phi} E t}-\frac{P R_{\phi}^{2}}{E t}\left(1-\frac{1}{9} \Delta^{2} q_{t}^{4}\right)\left(\frac{\dot{u}}{R_{\phi}}+\frac{2 q_{t}}{L}\right)\right)\right\} \mathrm{d} z
\end{aligned}
$$

According to the tenets of stability theory, the potential energy, $\Pi$, must be stationary at an equilibrium point, that is, $\delta \Pi=0$ for arbitrary $\delta w_{i}$ and $\delta u$. When integration by parts is performed on Eq. (23), ODEs are obtained, including three fourth-order ODEs associated with each $w_{i}(i=0,1,2)$ and one second-order ODE associated with $u$. Additionally, we minimize $\Pi$ with respect to the two parameters $q_{s}$ and $q_{t}$ and give rise to two integral constraints. (established in Appendix C)

\section{Numerical solution method}

The governing equations belong to the family of non-autonomous fourth-order ordinary differential boundary value problems with integral constraints. The numerical solution to these equations can be achieved by using the continuation package AUTO-07P [39]. The ability of this software to evaluate the location of bifurcation points is well known and is employed to determine the limit points and buckling behavior as discussed in the next section.

Here we try to solve the buckling governing equations based on half of the nanotube length arising from its symmetry along the longitudinal direction. The numerical solution can thus be acquired starting from the initial non-deformed state, $\left(w_{n}\right)_{0}=(u)_{0}=(M)_{0}=\left(q_{t}\right)_{0}=\left(q_{s}\right)_{0}=0$, through the continuous change of free parameters. Note that compared to other methods, this method does not need the introduction of any initial imperfections or any phenomenological assumptions. Key features such as the wavelength of buckling on the compressed side develop gradually 
during evolution of the system from the undeformed state rather than in a more abrupt way.

\section{Results and discussion}

\subsection{Equilibrium paths}

Numerical solutions for a $(5,5)$ SWCNT $(L=7.5 \mathrm{~nm})$ are acquired by solving the nonlinear governing equations. The relationship between the dimensionless moment and curvature $(m-\alpha)$ is shown in Fig. 3, which includes: (1) a nonlinear equilibrium path (blue solid line) under non-uniform ovalization deformation acquired by solving governing equations; (2) a nonlinear equilibrium path (red solid line) under uniform ovalization deformation acquired by Reissner's theory; (3) an equilibrium path (black solid line) acquired by the linear buckling theory.

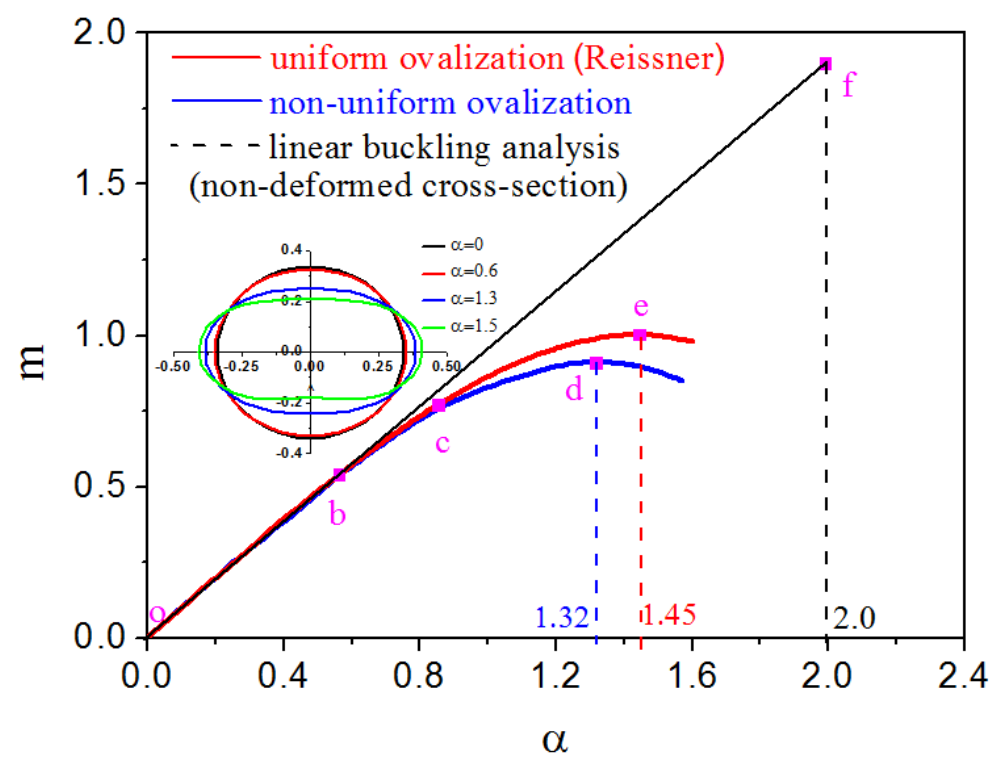

Fig. 3. Equilibrium paths and sectional ovalization deformation of a $(5,5)$ SWCNT $(L=7.5 \mathrm{~nm})$ under bending.

As seen from Fig. 3, point $\mathrm{b}(\alpha=0.58)$ is the separation point of linear and nonlinear equilibrium paths. In section ob, the equilibrium paths are approximately linear, and the sectional ovalization effect is negligible. Starting from point $b$, the nonlinear equilibrium paths depart from the linear one, and the degree of sectional ovalization is intensified compared to the linear buckling analysis, which results in a decrease of the bending stiffness of the SWCNT. The nonlinear equilibrium paths are related to the well-known Brazier effect [28], and the destiffening is due to the action of longitudinal stress on the curvature of bent CNTs. The resulting transverse pressure 
is directed towards the CNT neutral axis. This pressure leads both the top (compressive) and bottom (tensile) zones to move towards the neutral axis, thus resulting in the CNT ovalization. With further increase of curvature, the nonlinear equilibrium paths reach limit points first, corresponding to non-uniform and uniform ovalization limit points $\mathrm{d}$ ( $\alpha=1.32$ ) and e ( $\alpha=1.45)$, respectively. Compared to the linear critical buckling point $\mathrm{f}\left(k_{c}=\frac{t}{R^{2} \sqrt{3\left(1-v^{2}\right)}}, \alpha=2\right)$ [40], the critical curvature decreases by about $30 \%$ when sectional ovalization deformation is taken in account. When the curvature exceeds the limit point, the bearing capacity of the bent SWCNT drops abruptly, and finally it loses strength completely.

The sectional ovalization effect of CNTs during the bending process has already been demonstrated by numerous previous reports [35]. From the perspective of atomic structure, these transitions can be explained in terms of a model based on curvature-induced lattice mismatch [41]. Meanwhile, the radial deformation of CNTs strongly affects their physical and structural properties. For instance, it may cause semiconductor-metal transition, optical response change, and magnetic moment quenching in nanotubes [42], [43].

Here we define point $\mathrm{c}(\alpha=0.8)$ as the separation point of the non-uniform and uniform ovalization equilibrium paths. When the curvature is relatively small (section oc), uniform ovalization deformation occurs in the bent SWCNT, that is, the degree of ovalization is uniform along the nanotube length. After point $\mathrm{c}$, the nonlinear equilibrium path under non-uniform ovalization deformation departs from that under uniform ovalization deformation, and a significant increase of the local radial displacement $w_{n}$ results in an obvious non-uniform ovalization effect. As known from formula $(x+\varsigma+w \sin \varphi, y+\eta-w \cos \varphi), w_{n}$ can intensify the local sectional ovalization effect, thus leading to reduced bending stiffness of the SWCNT compared to the case under uniform ovalization. Therefore, the curvature corresponding to the ovalization limit point decreases from 1.45 to 1.32 , and the bearing capacity decreases by $10 \%$. This result also confirms, at least indirectly, that Reissner's theory with the assumption of uniform ovalization deformation overestimates the bearing capacity of the SWCNT. In addition, it is known from Eq. (4) that the last item, $\frac{1}{2} \dot{w}^{2}$, is always 
positive, which indicates that $w_{n}$ can hinder the increase of compressive strain during the bending process. Therefore, the model constructed in Section 2 may be used to describe the strain release of the SWCNT owing to local instability, which also induces the change of the strain energy mechanics as will be discussed in detail in Section 4.2.

\subsection{Instability mechanics}

A number of simulations and experiments have shown that CNTs are apt to buckle, during which ripples or kinks gradually occur from smooth bending, and the strain energy scales from harmonic to anharmonic. The bending process of a SWCNT can thus be divided into three stages by using an elastica model with a non-convex bending energy, i.e., low-curvature stage, mixed-curvature stage and high- curvature stage. In this sense, the SWCNT instability mechanics and the relationship between ripples and kinks can be elucidated in-depth by exploring phase transitions.

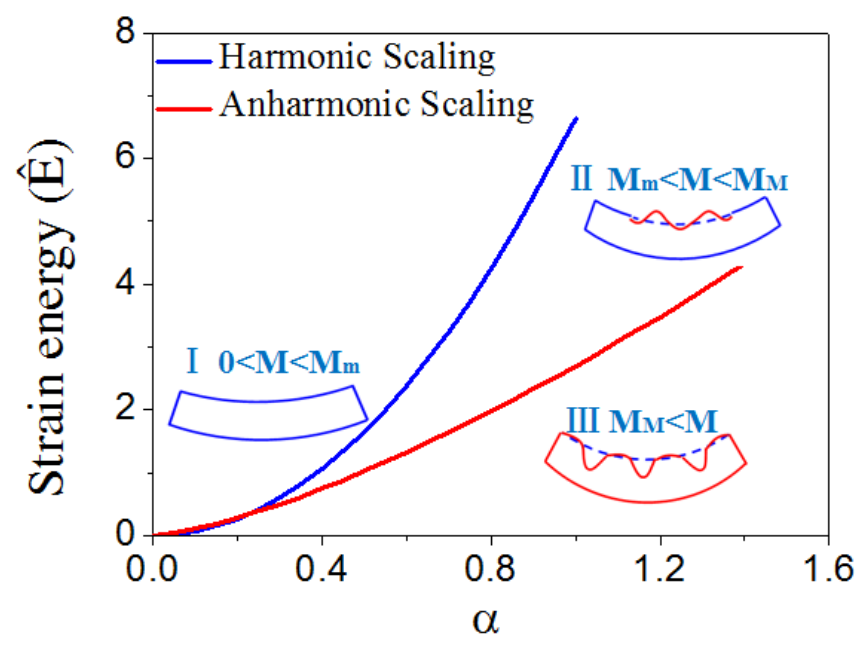

Fig. 4. Relationship between the strain energy $\hat{E}$ and curvature $\alpha$.

Fig. 4 depicts the relationship between the dimensionless strain energy $(\hat{E})$ and curvature $(\alpha)$ of a SWCNT. As seen from the figure, harmonic deformation occurs on the SWCNT under a small curvature, the strain energy scales harmonically, and a quadratic relationship is witnessed between the energy and curvature, as shown in Fig. 4 (blue line), which is in accord with the classical elastica theory as well as numerous MD simulation results [13], [44]:

$$
\hat{E}=\frac{1}{2} Q \alpha^{2}
$$


where the direct proportion coefficient $Q$ is the dimensionless bending stiffness. In this harmonic scaling, the hexagonal carbon rings on the SWCNT surface are slightly strained, while the whole carbon network is maintained. When the curvature exceeds a critical value, the slope becomes discontinuous, obvious non-uniform ovalization deformation occurs with ripples formed on the SWCNT surface, the strain energy scales from harmonic to anharmonic, and the scaling of the strain energy dramatically changes its nature to a robust power-law, as shown in Fig. 4 (red line):

$$
\hat{E}=S \alpha^{a} \quad(1<a<2)
$$

where $S$ is the coefficient, and $a$ is the exponent. Note that in this paper, the exponent characterizes an anharmonic elastic response in that it lies between the harmonic exponent of 2 and the typical exponent of 1 predicated by linearized buckling theory, which predicts that the structure can be deformed at a constant force. Since the analytical solutions to $S$ and $a$ are rather difficult to find, here we employ numerical solutions to obtain the fitted values $Q=6.64, S=2.7$, and $a=1.2$.

According to the non-convex curvature energy model proposed by Arroyo and Arias [45], here we define a new dimensionless energy form as:

$$
\Lambda(\alpha)=\min \left\{\frac{1}{2} Q \alpha^{2} ; S \alpha^{a}\right\}
$$

It is well known [46] that such a non-convex energy can lead to stressed phase mixtures. The bending of a SWCNT includes two phases, i.e., high- and low-strain phases. In the low-strain phase (LP), the bending of the SWCNT is smooth, and a quadratic relationship is observed between the energy and curvature. In the high-strain phase (HP), however, the degree of sectional ovalization is intensified with ripples formed on the SWCNT surface, and a power exponent relationship is witnessed between the energy and curvature. Indeed, upon the action of a bending moment $m$, the potential energy of the system $\Omega$ becomes:

$$
\Omega(\alpha, m)=\Lambda(\alpha)-m \alpha
$$

The bias introduced by loading produces the emergence of a new local energy minimum. Here we respectively define the bending moment, $M_{m}$ and $M_{M}$, at which the high-strain phase is created and the low-strain phase is destroyed. Therefore, for $M_{m}<m<M_{M}$ both the low-strain and high-strain phases can exist, which are 
shown in Fig. 4. Note that, the moment at which the energy levels of the two energy minima coincide is called the Maxwell moment $M_{0}$.

According to the theory proposed by Abeyaratne and Knowles [46], the necessary condition for the existence of energy wells is $\frac{\partial}{\partial k} \Omega(\alpha, m)=0$. Therefore, the two candidate minima can be expressed as $k_{L p}(m)$ and $k_{H p}(m)$. To assess whether these minima for energy wells are indeed possible, we define the curvature $k_{d}$ at which the harmonic and the anharmonic regimes meet:

$$
k_{d}=\left(\frac{2 S}{Q}\right)^{1 /(2-a)}
$$

The low-strain phase is possible if and only if $k_{L p} \leq k_{d}$, which leads to:

$$
M \leq Q\left(\frac{2 S}{Q}\right)^{1 /(2-a)}=M_{M}
$$

On the other hand, the high-strain phase is possible if and only if $k_{H p} \geq k_{d}$, hence:

$$
M \geq a S\left(\frac{2 S}{Q}\right)^{(a-1) /(2-a)}=M_{m}
$$

Thus, the Maxwell bending moment can be expressed as:

$$
M_{0}=\left[\frac{a}{2 Q(a-1)}(a S)^{1 /(a-1)}\right]^{(a-1) /(2-a)}
$$

By substituting $Q, S$ and $a$ in Eqs. (39)-(41), we can obtain the bending moment $M_{0}=0.6, M_{m}=0.55$ and $M_{M}=0.88$. According to the equilibrium paths depicted in Fig. 3, we find that $M_{m}$ is close to the moment corresponding to point b (separation point of linear/nonlinear equilibrium paths), while $M_{M}$ is close to the moment corresponding to point $\mathrm{d}$ (ovalization limit point), which are basically in agreement with the law disclosed in Section 4.1. When $m<M_{m}$, only the low-strain phase exists, and the SWCNT undergoes smooth bending. When $M_{m}<m<M_{M}$, the high-strain phase characterized by the sectional ovalization effect and ripple deformation occurs, although the low-strain phase is still present. Therefore, the SWCNT at this stage exhibits a so-called mixed-curvature stage with a combination of smooth bending, sectional ovalization effect and ripple deformation. With the 
increase of the in-plane strain energy, the SWCNT needs another means to release it. Since the in-plane stiffness in the SWCNT is much higher than the out-of-plane stiffness, the ripple deformation represents an effective configurational transition to release the in-plane strain energy, which leads to an energetically favorable state at sufficiently high bending (Eq. (15)). As such, the in-plane strain energy release is the driving force for ripples. When $m>M_{M}$, the low-strain phase disappears, and the high-strain phase is dominant, that is, ripples spread on the whole SWCNT surface and evolve along the depth direction to form kinks, resulting in reduced energy.

To describe the evolution mechanism from ripples to kinks, we present the relationship between the wave number $N$ (crest + trough) of $w_{n}$ and $\alpha$ in Fig. 5(a) When $\alpha>0.8$ (point $\mathrm{c}$ in Fig. 3), both $w_{0}$ and $w_{2}$ exhibit an obvious corrugation change, with the former having 3 crests and 2 troughs $(3,2)$ and the latter having 4 crests and 3 troughs $(4,3)$, while $w_{1}$ remains stable, as shown in Fig. 6(a). Compared with the other two modes, the axisymmetric deformation mode $\left(w_{0}\right)$ acts as a dominant role owing to its larger amplitude. It can be deduced from the formula, $w(z, \varphi)=\sum_{n=0}^{2} w_{n}(z) \cos n \varphi$, that $w_{0}, w_{1}$ and $w_{2}$ together lead to a non-uniform ovalization deformation, resulting in ripples on the central region of the SWCNT surface and a step change of the wave number. When the moment increases to the ovalization limit point (d), the wave number of $w_{0}$ and $w_{1}$ changes by one, while that of $w_{2}$ remains unchanged. Under the joint effect of $w_{0}, w_{1}$ and $w_{2}$, ripples spread to both ends of the SWCNT, and the wave number increases from 5 to 7 , as shown in Fig. 6(b). At this stage ripples occupy the whole SWCNT surface, and reach the maximum number of this size, that is, the high-strain phase is fully dominant. If the moment further increases, the wave number remains constant, while the waveform has a significant degree of change. We define $\xi$ as the changing degree of waveform, which can be expressed as follows:

$$
\xi=\frac{1}{n} \sum_{i=1}^{n} \frac{A_{m i}}{\lambda_{i}} \times 100 \%
$$

where $A_{m i}$ is the amplitude and $\lambda_{i}$ is the wavelength of each waveform indexed by $i$. As shown in Fig. 5(b), $\xi$ increases with the increase of curvature along the L1 
direction between the point $\mathrm{c}$ and $\mathrm{d}$. When the moment exceeds the ovalization limit point (d), $\xi$ exhibits a step change and increases along the L2 direction. Note that the slope of L2 is much higher than that of L1, indicating that the changing degree of amplitude is far higher than that of wavelength. Thereafter, the wavelength of ripples stays unchanged while the amplitude increases steadily. In other words, the waveform is enlarged along the amplitude direction, and ripples evolve into kinks gradually. From this point of view, ripples can be regarded as the original form of kinks. Thus, the numerical results suggest that the ripple deformation mode is an efficient one and allows the system to tunnel between smoothly bent harmonic states and kink states. It is worth noting that $\xi$ stays below 0.25 in the mixed-curvature stage, suggesting that the amplitude of ripples is small compared to the wavelength, and these tiny corrugations are rather hard to observe.
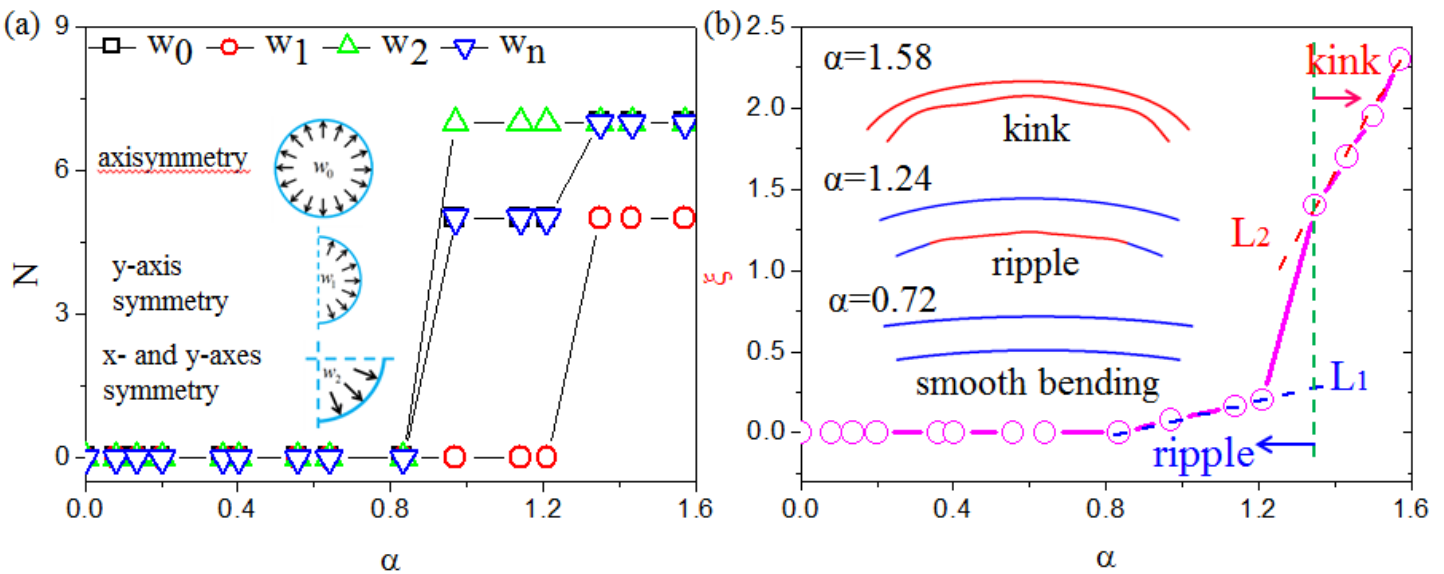

Fig. 5. (a) Relationship between the wave number $N$ (crest + trough) of $w_{n}$ and $\alpha$; (b) Relationship between the degree of waveform $\xi$ and $\alpha$.
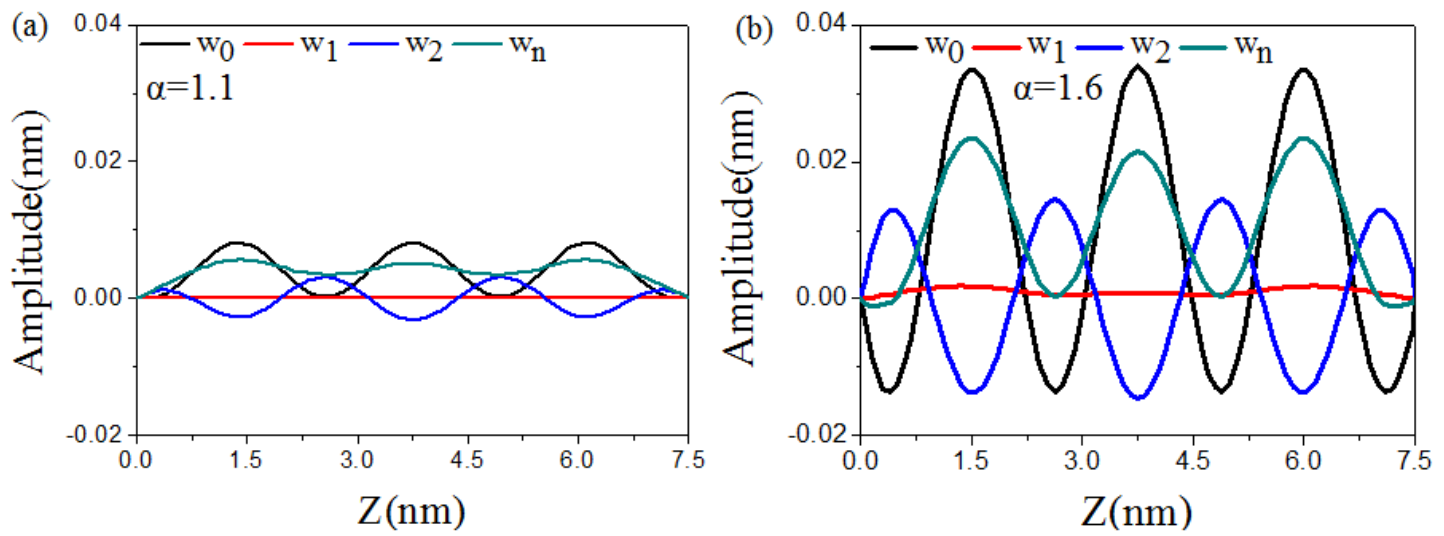

Fig. 6. Waveform of the different modes $w_{n}$ : (a) Waveform of $w_{n}$ at $\alpha=1.1$; (b) Waveform of $w_{n}$ at $\alpha=1.6$. 


\subsection{OMD simulation}

In the next part of this study, the buckling behavior of a SWCNT under bending is simulated by using the OMD method [47]. In contrast with the traditional molecular dynamics whose loading mode includes the application of a bending angle by fixing the atoms at each end, followed by the release of the axial strain, we propose here so-called objective boundaries where the bending angle is only applied on the simulation unit cells while the internal atoms can realize free motion without any restriction. The advantage of our method lies in the fact that it does not need the release of the axial strain and the final curvature and configuration of the SWCNT are the results after relaxation, which is closer to the actual bending situation.

When using PBC along one dimension, the positions $X_{j}$ of the $n$ atoms located in the simulation unit cells or supercells are replicated with

$$
X_{j, \zeta}=\zeta T+X_{j} \quad j=1, \ldots, n
$$

where $X_{j, \zeta}$ labels the position of atom $j$ located in the replica $\zeta$ of the initial cell and $T$ is the translational vector. If the structural parameter $|T|$ is varied around its optimized value, pure axial stress states are introduced. When using objective boundaries for bending, we consider in the simulation the same $n$ atoms but the repetition rule is changed from translation rotation, with

$$
X_{j, \zeta}=\Gamma^{\zeta} X_{j} \quad j=1, \ldots, n
$$

This relationship indicates that the $n$ atoms located in the $\zeta$ cell are obtained from the ones located in the original cell by repeated rotations of angle $\theta$ around an axis perpendicular onto the CNT axis, as prescribed by the rotational matrix $\Gamma$. In the simulations reported here the potential is short-range so we considered only the $\zeta= \pm 1$ replica

We carry out investigations on $(5,5)$ and $(15,15)$ SWCNTs. In both cases, the length of the objective simulation unit cell is selected to be $2.5 \mathrm{~nm}$. This cell is larger than the minimum cell as we would like to capture not only ovalization but also the kinking process. Various degrees of bending are applied to the simulation unit cell by varying $\theta$ in steps of at most $0.04^{\circ}$ per nm of nanotube length. At each bending increment step OMD is performed at $300 \mathrm{~K}$ for 500 time steps of $2 \mathrm{fs}$ each, followed 
by conjugate gradient energy minimization. This protocol was previously found to be effective in obtaining the minimum energy configuration under the constraints Eq. (18) [13]. This is important as we would like to identify the threshold for the onset of rippling.

Fig. 7(a)-(b) depicts the relationship between strain energy and moment vs bending angle of $(5,5)$ and $(15,15)$ simulation unit cells. In accord with what is analyzed in Section 4.2, the moment drops abruptly indicating the formation of kinks when the curvature reaches a critical value corresponding to the ovalization limit point (d) shown in Fig. 3. Meanwhile, the SWCNT undergoes smooth bending under a small curvature, as shown in Fig. 7(c)-(d). Under a small curvature, its top and bottom sides undergo stretching and compression, respectively. The initial curvature of a CNT deforms the lattice from a stress-free planar hexagonal graphene structure to a wrapped structure containing an initial internal stress. The hexagonal rings on the tube surface are only slightly strained and the hexagonal carbon network is maintained. When the curvature reaches a critical value, ripples are progressively formed and symmetrically distributed along the nanotube bottom surface, through which the strain energy on the compressed sides is released. These changes increase the surface area of the bending side and introduce an effective lattice mismatch [16]. When the curvature is large, the bottom carbon atoms move along the amplitude direction and evolve into deep kinks. Due to strong covalent binding, the flattening of the nanotube cross section at the buckling site occurs in a gradual way. However, even up to the formation of kinks, the structure remains all-hexagonal without any topological defects. Compared to the thinner $(5,5)$ SWCNT, the thicker $(15,15)$ SWCNT needs a higher degree of deformation at the cross section to form complete kinks. As such, $\eta$ corresponding to the ripples of the thicker $(15,15)$ SWCNT is higher, and is easier to observe, as shown in Fig. 7(d). 


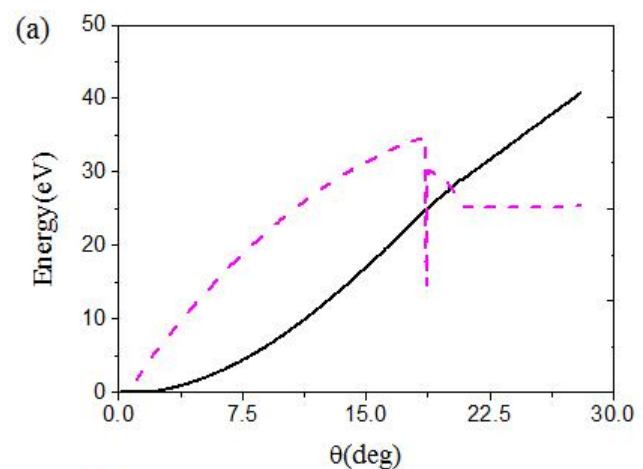

(c)
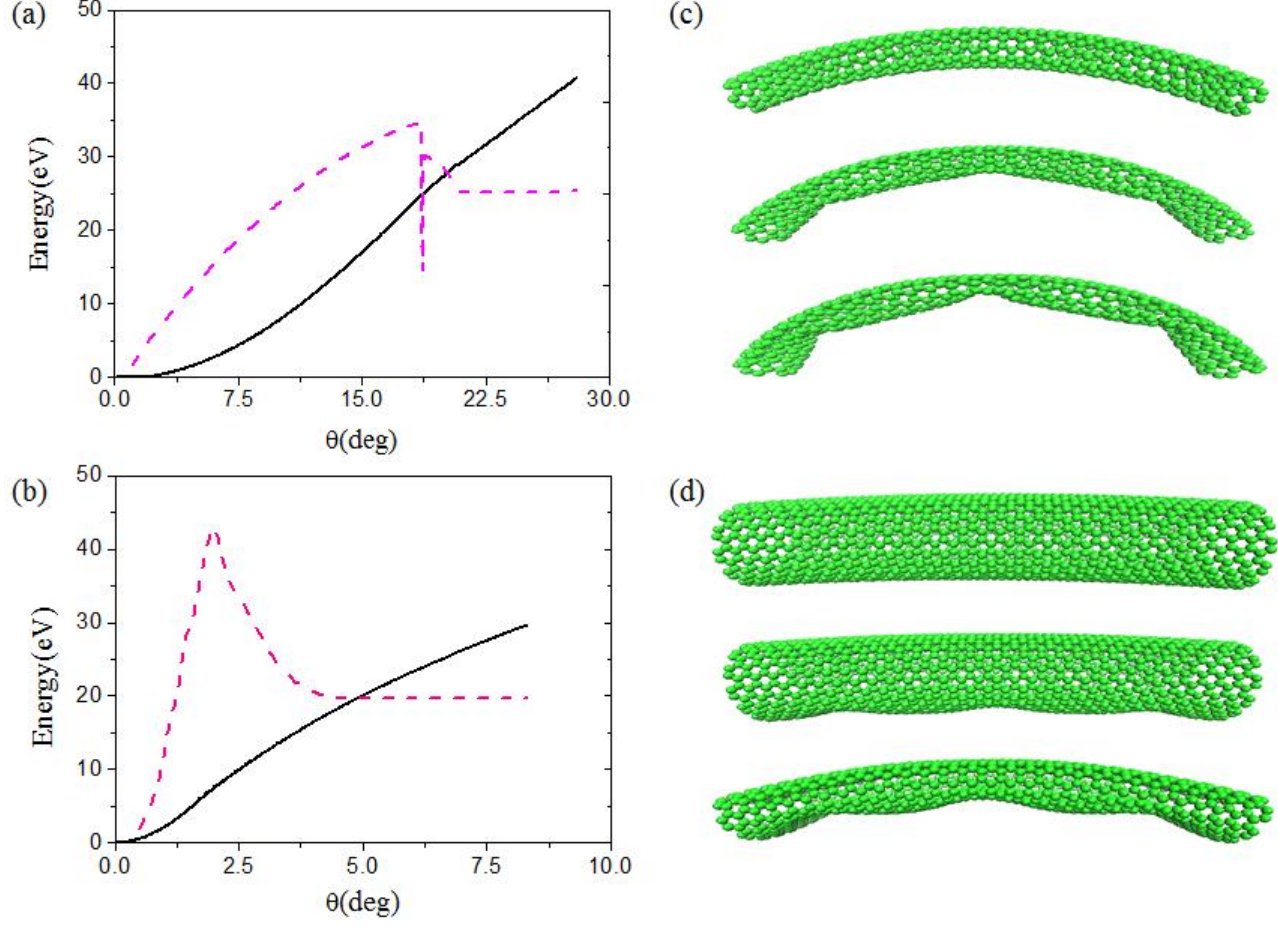

(d)
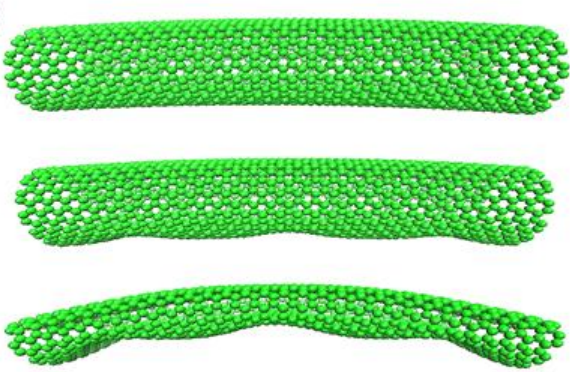

Fig. 7. Strain energy and morphologies of two SWCNTs under bending: (a) Relationship between the strain energy (black continuous line) and bending moment (pink dashed line) vs bending angle $\theta$ for $(5,5)$ SWCNT simulation cell; (b) Relationship between the strain energy (black continuous line) and bending moment (pink dashed line) vs bending angle $\theta$ for $(15,15)$ SWCNT simulation cell; (c) Morphologies under different $\alpha(0.73,1.36,1.62)$ for $(5,5)$ SWCNT; (d) Morphologies under different $\alpha(0.14,0.18,0.86)$ for $(15,15)$ SWCNT.

\subsection{Size effect}

We define the point where the moment starts to drop as the critical curvature $\left(K_{c r}\right)$, while Reissner's theory essentially says that the tube should buckle when the bending moment reaches its maximum. Fig. 8(a) shows the relationship between the SWCNT radius and critical curvature. Both the results of numerical calculation and OMD simulation prove that a SWCNT with a smaller radius is more difficult to be buckled than that with a larger radius. Moreover, the critical curvature is approximately in inverse proportion to the square of tube radius (red dashed line), that is, $k_{c} \infty 1 / 4 R^{2}$, which is in good agreement with the previous reports [48]. Comparing the results of numerical calculation and OMD simulation, we find that the critical curvature obtained by numerical calculation is slightly higher than that obtained by OMD simulation. The difference between the two reaches its maximum of ca. $12 \%$ at $R=0.339 \mathrm{~nm}$, and decreases with the increase of the SWCNT radius. The reason for 
this minor difference lies in different assumptions adopted in numerical calculation and OMD simulation: the OMD simulation takes into account the geometric imperfection of the SWCNT (a discrete structure), while continuum mechanics regards it as perfect (a continuum structure). It is well known that buckling is rather sensitive to geometric imperfection, which may induce a decrease of the critical buckling load. As such, the critical curvature obtained by numerical calculation is slightly higher than that obtained by OMD simulation, which is in accordance with what has been reported before [48].

Fig. 8(b) reveals the relationship between the aspect ratio $(L / R)$ and critical curvature. As seen from the figure, the aspect ratio for a long SWCNT $(L / R>10)$ only affects the ripple number, and has little, if any, impact on the critical curvature. For a short SWCNT $(L / R<10)$, the critical curvature decreases with the decrease of the aspect ratio when it reaches a certain threshold. It has been reported elsewhere that this threshold is intimately related to the SWCNT radius while being independent of its chirality [40] However, we will not lay too much emphasis on this aspect since it is not the key point of our current study.
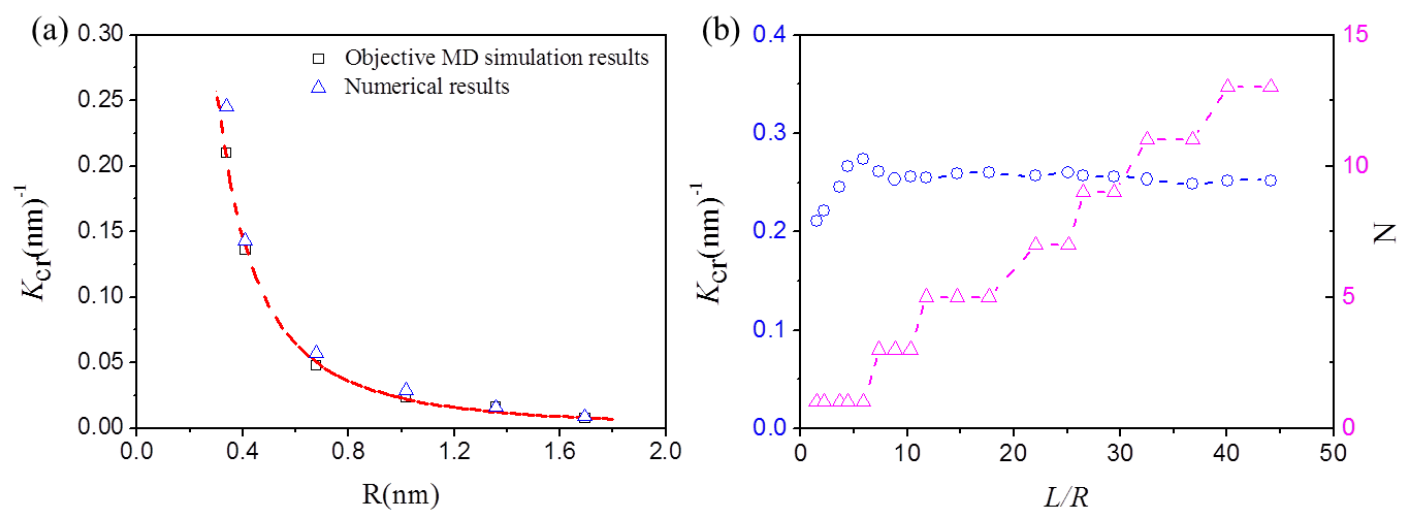

Fig. 8(a) Relationship between the SWCNT radius and critical curvature $K_{c r}$; (b) Relationship between the aspect ratio $(L / R)$ and critical curvature $K_{c r}$.

\subsection{Effect of internal pressure}

As is well known, CNTs are good media for storing hydrogen, oxygen, metals and metal oxides. In this paper, hydrogen storage is used as a case for exploring the effect of internal pressure. The impact of hydrogen molecules on the nanotube wall is described by the L-J potential, which is equivalent to the internal pressure [49]: 


$$
P=-\frac{1}{6 V_{o l}} \sum_{j(\neq i)} r_{i j} \frac{d V_{L J}}{d r_{i j}}
$$

where $V_{o l}$ is the average volume of particles, and is $V_{o l}=\frac{\sqrt{2}}{2} d_{\mathrm{H}_{2}-\mathrm{H}_{2}}^{3}$ since the hydrogen atoms obey $\mathrm{AB}$ stacking. $d_{\mathrm{H}_{2}-\mathrm{H}_{2}}$ is the distance between two hydrogen molecules. Therefore, the relationship between the equivalent pressure $P$ and $d_{\mathrm{H}_{2}-\mathrm{H}_{2}}$ can be expressed as follows, where $\varepsilon_{\mathrm{H}_{2}-\mathrm{H}_{2}}$ and $\sigma_{\mathrm{H}_{2}-\mathrm{H}_{2}}$ are the potential well depth and van der Waals separation distance of $\mathrm{H}_{2}-\mathrm{H} 2$, respectively.

$$
P=\frac{48 \sqrt{2} \varepsilon_{\mathrm{H}_{2}-\mathrm{H}_{2}}}{\sigma_{\mathrm{H}_{2}-\mathrm{H}_{2}}^{3}}\left[2\left(\frac{\sigma_{\mathrm{H}_{2}-\mathrm{H}_{2}}}{d_{\mathrm{H}_{2}-\mathrm{H}_{2}}}\right)^{15}-\left(\frac{\sigma_{\mathrm{H}_{2}-\mathrm{H}_{2}}}{d_{\mathrm{H}_{2}-\mathrm{H}_{2}}}\right)^{9}\right]
$$

Jiang et al. [50] has built up a cohesive law between a CNT and polymer molecules based on van der Waals interactions. By employing this law while replacing polymer molecules by hydrogen molecules, we can acquire the relationship between $P$ and $d_{C-H_{2}}$, where $\varepsilon_{{\mathrm{C}-\mathrm{H}_{2}}}$ and $\sigma_{\mathrm{C}-\mathrm{H}_{2}}$ are the potential well depth and van der Waals separation distance of $\mathrm{C}-\mathrm{H} 2$, respectively.

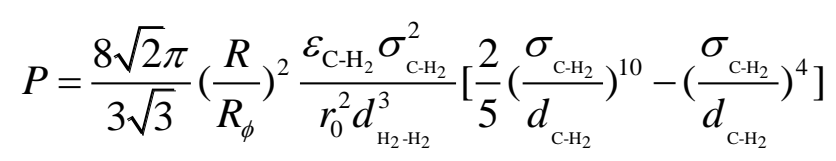

Considering such factors as expansion by hydrogen storage and $\mathrm{C}-\mathrm{H}_{2}$ distance, actually hydrogen can only be stored in a circular tube with a radius of $R_{\phi}-d_{\mathrm{C}-\mathrm{H}_{2}}$. Therefore, hydrogen stored per unit length is $\pi\left(R_{\phi}-d_{C-H_{2}}\right)^{2} V_{o l}^{-1}$. The hydrogen storage capacity of the SWCNT per unit volume can be expressed as:

$$
N_{\mathrm{H}}=\frac{\pi\left(R_{\phi}-d_{{\mathrm{C}-\mathrm{H}_{2}}}\right)^{2} V_{o l}^{-1}}{\pi R_{\phi}^{2}}=\left(\frac{R_{\phi}-d_{{\mathrm{C}-\mathrm{H}_{2}}_{2}}}{R_{\phi}}\right)^{2} \frac{\sqrt{2}}{d_{\mathrm{H}_{2}-\mathrm{H}_{2}}^{3}}
$$

The relationship between the hydrogen storage capacity $N_{\mathrm{H}}, \mathrm{C}-\mathrm{H}_{2}$ distance $d_{C-\mathrm{H}_{2}}, \mathrm{H}_{2}-\mathrm{H}_{2}$ distance $d_{\mathrm{H}_{2}-\mathrm{H}_{2}}$ and internal pressure $P$ is summarized in Fig. 9(a). It can be seen from Fig. 9(a) that increasing $N_{\mathrm{H}}$ leads to decreasing $d_{C-\mathrm{H}_{2}}$ and $d_{\mathrm{H}_{2}-\mathrm{H}_{2}}$, which finally approach 0.24 and $0.19 \mathrm{~nm}$, respectively. Note that decreasing $d_{C-\mathrm{H}_{2}}$ 
and $d_{\mathrm{H}_{2}-\mathrm{H}_{2}}$ result in increasing van der Waals interactions. Therefore, the internal pressure discussed in this paper is restricted to a range of 0-70 GPa.
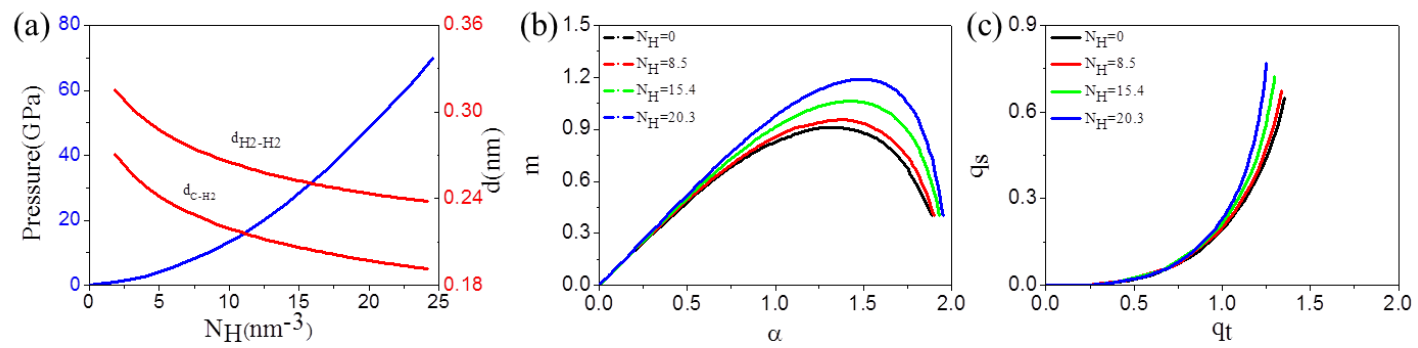

Fig. 9. (a) Relationship between the hydrogen storage capacity $N_{\mathrm{H}}, \mathrm{C}-\mathrm{H}_{2}$ distance $d_{C-\mathrm{H}_{2}}, \mathrm{H}_{2}-\mathrm{H}_{2}$ distance $d_{\mathrm{H}_{2}-\mathrm{H}_{2}}$ and internal pressure $P$; (b) Relationship between the dimensionless moment and curvature $(m-\alpha)$ under different hydrogen storage capacities; (c) Relationship between $q_{s}$ and $q_{t}$ under different hydrogen storage capacities.

By substituting Eq. (48) in Eqs. (24)-(31), we can obtain the relationship between the dimensionless moment and curvature $(m-\alpha)$ under different hydrogen storage capacities, as shown in Fig. 9(b). It can be seen that the internal pressure increases from 0 to $50 \mathrm{GPa}$ when the hydrogen storage capacity increases from 0 to $20.3 \mathrm{~nm}^{-3}$. In response, the moment corresponding to the ovalization limit point increases by $33 \%$, and the curvature increases by $16 \%$. As can be seen from Eq. (19), the internal pressure can raise the internal resisting moment, enhance the tangent bending stiffness, and improve the bearing capacity of the SWCNT when it acts on the curvature. Meanwhile, in the small curvature range, the equilibrium path remains approximately linear with a higher hydrogen storage capacity and the non-linear effect is not obvious. This is because the higher the hydrogen storage capacity, the higher the internal pressure. The distances between the hydrogen molecules and the wall of the SWCNT become shorter when large quantities of hydrogen molecules are stored in the SWCNT. When the sectional ovalization deformation occurs, the van der Waals interactions between $\mathrm{H}_{2}$ and $\mathrm{C}-\mathrm{H}_{2}$ are enhanced, by which the shrinkage tendency of the nanotube wall is hindered. Therefore, the sectional ovalization effect is weakened, and the decrease of the bending stiffness is alleviated. In addition, as seen in Fig. 9(c), the internal pressure also has an impact on enhancing the shear stiffness. When $q_{s}$ is fixed, the higher the internal pressure, the lower the $q_{t}$. We can deduce from Eq. (19) that the internal pressure directly increases the beam shear 
stiffness. This result is identical to that derived independently by Fichter using shell theory [51].

Table 1. Parameters obtained computationally for several internal pressures.

\begin{tabular}{ccccccc}
\hline$P(\mathrm{GPa})$ & $N_{H}\left(\mathrm{~nm}^{-3}\right)$ & $Q$ & $S$ & $a$ & $M_{m}$ & $M_{M}$ \\
\hline 0 & 0 & 6.64 & 2.7 & 1.2 & 0.55 & 0.88 \\
10 & 8.5 & 10.16 & 3.0 & 1.2 & 0.54 & 0.90 \\
30 & 15.4 & 18.77 & 3.5 & 1.2 & 0.56 & 0.94 \\
50 & 20.3 & 27.00 & 3.77 & 1.2 & 0.57 & 0.97 \\
\hline
\end{tabular}

Table 1 summarizes the relationship between hydrogen storage capacity and $P, Q$, $S, a, M_{m}$ and $M_{M}$. The increase of internal pressure significantly increases the value of $Q$ since it mainly affects the bending stiffness, while it exerts a negligible effect on the value of $a$. On the other hand, with the increase of hydrogen storage capacity and $P, M_{m}$ remains nearly unchanged while $M_{M}$ increases significantly. When $P$ increases from 0 to $50 \mathrm{GPa}$, the range of the mixed-curvature stage $\left(M_{M}-M_{m}\right)$ increases by $33 \%$, indicating that the threshold into the third stage (high-curvature stage) is raised. In other words, the SWCNT is inclined to stay at the mixed-curvature stage. This result confirms the fact that the sectional ovalization effect and ripples more easily occur in a MWCNT or a SWCNT whose interior is adsorbed with substances. From the structural perspective, the internal substances hinder the evolution of ripples into kinks as amplitude increases. From the non-convex energy point of view, the presence of internal substances is equivalent to the application of internal pressure that prolongs the mixed-strain stage stage, thus making ripples increase along the $\mathrm{L} 1$ direction. Ripples are much easier to observe in this case.

\section{Conclusion}

In this paper, we ascribe the buckling behavior of CNTs under bending to the fourth-order nonlinear ordinary differential equations (buckling governing equations) with integral conditions, and solved them by using a continuation algorithm. The formation and evolution mechanics of CNT buckling instability have been explored, and the buckling behavior of CNTs has also been analyzed by OMD simulation in 
order to validate the former numerical results. Our method does not need any assumption on the wavelength or wave number, and is therefore more accurate in approaching the actual formation and evolution mechanics of ripples and kinks (CNT buckling instability) compared to other methods. The main conclusions are listed as follows:

1. There exist low- and high-strain phases during the bending process of CNTs, which make the strain energy scale from harmonic to anharmonic. The whole bending process can thus be divided into three stages based on two critical values $\left(M_{m}\right.$ and $\left.M_{M}\right)$ : a low-curvature stage characterized by smooth bending, a mixed-curvature stage characterized by a combination of smooth bending, sectional Brazier effect and surface ripples, and high-curvature stage characterized by kinks. According to the relationship between $w_{n}, \xi$ and $\alpha$, we conclude that ripples are formed from the central part of CNTs, and then spread to both ends. When ripples occupy the whole CNT surfaces, they start to evolve along the amplitude direction and eventually develop into kinks.

2. The sectional ovalization effect and ripples reduce the bending stiffness of CNTs, thereby reducing their bearing capacity. When the aspect ratio of CNTs is high enough $(L / R>10)$, it only affects the ripple number, and has little, if any, impact on the critical curvature. On the other hand, CNTs with a smaller radius are more difficult to be buckled compared to those with a larger radius, and the critical curvature is approximately in inverse proportion to the square of radius.

3. The internal pressure of hydrogen-charged CNTs can hinder the sectional ovalization deformation, thus enhancing the bending stiffness and transverse shear stiffness. Compared to SWCNTs, hydrogen-charged CNTs or MWCNTs have a longer mixed-strain stage due to the presence of internal pressure. When the internal pressure increases from 0 to $50 \mathrm{GPa}$, the range of mixed-curvature stage increases by about 33\%. Therefore, hydrogen-charged CNTs or MWCNTs are more inclined to exhibit a ripple configuration.

\section{Acknowledgements}

The authors gratefully acknowledge financial supports from National Natural Science Foundation of China, 11172079 and 11572099; Program for New Century 
Excellent Talents in University, NCET-11-0807; Natural Science Foundation of Heilongjiang Province of China, 40000045-6-15441; the Fundamental Research Funds for the Central Universities, HIT.BRETIII.201209. Meanwhile, we thank Dr. B. Li in Shanghai Jiao Tong University for his great help.

\section{Appendix A. Internal pressure effect}

In molecular mechanics, the interaction between carbon atoms can be described by the Tersoff or Brenner potential [52], [53]:

$$
V\left(r_{i j}, \theta_{i j k} ; k \neq i, j\right)=V_{R}\left(r_{i j}\right)-B_{i j} V_{A}\left(r_{i j}\right)
$$

where $V$ is the interatomic potential, $r_{i j}$ is the distance between atoms $i$ and $j, V_{R}(r)$ and $V_{A}(r)$ are the repulsive and attractive pair terms, and $B_{i j}$ is the milti-body coupling term.

$$
B_{i j}=\left[1+\sum_{k(\neq i, j)} G\left(\cos \theta_{i j k}\right) f_{c}\left(r_{i k}\right)\right]^{-0.5}
$$

The interaction between carbon and hydrogen atoms (or molecules) can be described by the classical Lennard-Jones 6-12 potential. According to the atomistic-based finite-deformation shell theory proposed by Huang et al. [54], the constitutive relationship of a CNT satisfies:

$$
\begin{gathered}
\left(\sigma_{\varphi}+\sigma_{z}\right) t=\frac{1}{\sqrt{3}}\left(\frac{\partial^{2} V}{\partial r_{i j}^{2}}\right)_{0}\left(\varepsilon_{\varphi}+\varepsilon_{z}\right) \\
\left(\sigma_{\varphi}-\sigma_{z}\right) t=\frac{B}{8 \sqrt{3}}\left(\varepsilon_{\varphi}+\varepsilon_{z}\right)
\end{gathered}
$$

where $\varepsilon_{\varphi}$ and $\varepsilon_{z}$ are the circumferential and axial strains, and $\sigma_{\varphi}$ and $\sigma_{z}$ are the corresponding stresses, the wall thickness $t=0.075 \mathrm{~nm}$ [34]. The subscript " 0 " is the state of the initial equilibrium position, that is, $\left(r_{i j}\right)_{0}=r_{0}=0.142 \mathrm{~nm}$ and $\left(\theta_{i j k}\right)_{0} \approx 120^{\circ}$, where $B$ can be expressed as:

$$
\begin{aligned}
& B=\frac{3(1-C)^{2}}{r_{0}^{2}}\left[4\left(\frac{\partial V}{\partial \cos \theta_{i j k}}\right)_{0}+6\left(\frac{\partial^{2} V}{\partial \cos _{i j k} \partial \cos _{i j k}}\right)_{0}-3\left(\frac{\partial^{2} V}{\partial \cos _{i j k} \partial \cos _{i j l}}\right)_{0}\right] \\
& +4(1+C)^{2}\left(\frac{\partial^{2} V}{\partial r_{i j}^{2}}\right)_{0}-\frac{12\left(1-C^{2}\right)}{r_{0}}\left(\frac{\partial^{2} V}{\partial r_{i j} \partial \cos _{i j k}}\right)_{0}
\end{aligned}
$$


and

$$
C=1-\frac{8 r_{0}^{2}\left(\frac{\partial^{2} V}{\partial r_{i j}^{2}}\right)_{0}+12 r_{0}\left(\frac{\partial^{2} V}{\partial r_{i j} \partial \cos _{i j k}}\right)_{0}}{12\left(\frac{\partial V}{\partial \cos \theta_{i j k}}\right)_{0}+4 r_{0}^{2}\left(\frac{\partial^{2} V}{\partial r_{i j}^{2}}\right)_{0}+18\left(\frac{\partial^{2} V}{\partial \cos _{i j k} \partial \cos _{i j k}}\right)_{0}-9\left(\frac{\partial^{2} V}{\partial \cos _{i j k} \partial \cos _{i j l}}\right)_{0}+12 r_{0}\left(\frac{\partial^{2} V}{\partial r_{i j} \partial \cos _{i j k}}\right)_{0}}
$$

From Eq. (7)-(8) we can obtain:

$$
E t=\frac{2}{\sqrt{3}\left(\frac{1}{\left(\partial^{2} V / \partial r_{i j}^{2}\right)_{0}}+\frac{8}{B}\right)}
$$

\section{Appendix B. Energetic formulation}

The total potential of the CNT, $\Pi$, is mainly composed of bending energy $U_{B}$, axial strain energy $U_{M}$, shear strain energy $U_{S}$, circumferential bending energy $U_{C}$, pressure potential $U_{P}$, and work done by load $U_{L}$.

$$
\Pi=U_{B}+U_{M}+U_{S}+U_{C}+U_{P}-U_{L}
$$

(1) Bending energy $U_{B}$

The bending energy of the CNT is the collective effect of global and local deformations, and can be expressed as:

$$
U_{B}=\frac{1}{2} E t \int_{0}^{L} \int_{0}^{2 \pi R_{\phi}}(\ddot{W}+\ddot{w})^{2}(y+\eta)^{2} \mathrm{~d} s \mathrm{~d} z
$$

where $(y+\eta)^{2} t \mathrm{~d} s$ is the second moment of area about the $\mathrm{x}$-axis, which takes into account the sectional ovalization deformation of the CNT under bending. If the sectional ovalization deformation is neglected, the secondary moment of area degenerates to $y^{2} t \mathrm{~d} s$, which is identical to Euler bending.

(2) Axial strain energy $U_{M}$

The final axial strain energy can be expressed as:

$$
U_{M}=\frac{1}{2} E t \int_{0}^{L} \int_{0}^{2 \pi R_{\phi}} \varepsilon^{2} \mathrm{~d} s \mathrm{~d} z
$$


(3) Shear strain energy $U_{S}$

The shear effect can be approached by using the Timoshenko beam model. Meanwhile, the additional effect imposed by the local deformation should also be taken into account, and the shear strain energy can be expressed as:

$$
U_{s}=\frac{1}{2} G t \int_{0}^{L} \int_{0}^{2 \pi R_{\phi}} \gamma_{y z}^{2} \mathrm{~d} s \mathrm{~d} z
$$

where the shear strain $\gamma_{y z}=\frac{\partial W}{\partial z}+\frac{\partial w}{\partial z}-\theta+\frac{\partial u_{\varphi}}{\partial y}$.

(4) Circumferential bending energy $U_{C}$

Considering the curvature $\partial^{2} w / \partial s^{2}$ induced by the local deformation, we can obtain the circumferential bending energy:

$$
U_{c}=\frac{1}{2} D \int_{0}^{L} \int_{0}^{2 \pi R_{\phi}}\left(\frac{\partial^{2} w}{\partial s^{2}}\right)^{2} \mathrm{~d} s \mathrm{~d} z=\frac{D}{2 R_{\phi}^{3}} \int_{0}^{L} \int_{0}^{2 \pi}\left(\frac{\partial^{2} w}{\partial \varphi^{2}}\right)^{2} \mathrm{~d} \varphi \mathrm{d} z
$$

where the bending stiffness $D=\frac{E t^{3}}{12\left(1-v^{2}\right)}$.

(5) Pressure potential $U_{P}$

If we assume the pressure is constant, then we mainly consider the effect of volume change induced by the pressure on the structural potential. According to the theory proposed by Davids and Zhang [55], the pressure potential can be expressed as follows when the sectional ovalization deformation of the CNT is taken into account:

$$
U_{P}=-P \Delta V_{v o l}=\int_{0}^{L}\left[\pi R_{\phi}^{2}\left(1-\frac{1}{9} \Delta^{2} q_{t}^{4}\right)\left(\frac{1}{2} P \gamma_{y z}^{2}+P R_{\phi}\left(\frac{\dot{u}}{R_{\phi}}+\frac{2 q_{t}}{L}\right)\right)\right] \mathrm{d} z
$$

(6) Work done by load $U_{L}$

The work done by load is the sum of the contributions from the internal and external moments. Each term is basically the moment multiplied by the local rotation, therefore the work done by load can be expressed as:

$$
U_{L}=\int_{0}^{L} \int_{0}^{2 \pi R_{\phi}} E t \varepsilon_{z} \dot{u}_{\varphi} \mathrm{d} s \mathrm{~d} z+\int_{0}^{L} M\left(\frac{\dot{u}}{R_{\phi}}+\frac{2 q_{t}}{L}\right) \mathrm{d} z
$$

\section{Appendix C. Governing equations}


Four governing equations are obtained by variation method and expressed as follows:

$$
\begin{aligned}
& R_{\phi}^{2}\left[\frac{1}{2}\left(2-\Delta q_{t}^{2}\right) \dddot{w}_{0}+\frac{1}{6}\left(3-2 \Delta q_{t}^{2}\right) \dddot{w}_{2}\right]-\left[3 \dot{w}_{0}^{2} \ddot{w}_{0}-\frac{q_{t} R_{\phi}}{2 L} \ddot{w}_{1}\left(4-\Delta q_{t}^{2}\right)+\frac{3}{4} \dot{w}_{1}\left(2 \ddot{w}_{1} \dot{w}_{2}+\dot{w}_{1} \ddot{w}_{2}\right.\right. \\
& \left.\left.+2 \ddot{w}_{0} \dot{w}_{1}+4 \dot{w}_{0} \ddot{w}_{1}\right)+\frac{3}{2} \dot{w}_{2}\left(\ddot{w}_{0} \dot{w}_{2}+2 \dot{w}_{0} \ddot{w}_{2}\right)+\left(\ddot{u}_{1}+\ddot{w}_{1}\right)\right]-\left[\frac{2 G}{E}+\frac{2 P R_{\phi}}{E t}\left(1-\frac{1}{9} \Delta^{2} q_{t}^{4}\right)\right]\left[\frac{\dot{u}}{r}\right. \\
& \left.+\frac{2\left(q_{s}-q_{t}\right)}{L}\right]=0
\end{aligned}
$$

$R_{\phi}^{2}\left[\frac{1}{12}\left(9-5 \Delta q_{t}^{2}\right) \dddot{w}_{1}\right]-\left[\frac{9}{8} \dot{w}_{1}^{2} \ddot{w}_{1}-\frac{q_{t} R_{\phi}}{2 L} \ddot{w}_{0}\left(4-\Delta q_{t}^{2}\right)-\frac{q_{t} R_{\phi}}{3 L} \ddot{w}_{2}\left(3-\Delta q_{t}^{2}\right)\right.$

$+\frac{3}{2}\left(\ddot{w}_{0} \dot{w}_{1} \dot{w}_{2}+\ddot{w}_{1} \dot{w}_{0} \dot{w}_{2}+\ddot{w}_{2} \dot{w}_{0} \dot{w}_{1}+2 \ddot{w}_{0} \dot{w}_{0} \dot{w}_{1}+\dot{w}_{0}^{2} \ddot{w}_{1}\right)+$

$\left.\frac{1}{2}\left[\ddot{u}\left(2 \dot{w}_{0}+\dot{w}_{2}\right)+\dot{u}\left(2 \ddot{w}_{0}+\ddot{w}_{2}\right)\right]+\frac{3}{4}\left(\ddot{w}_{1} \dot{w}_{2}^{2}+2 \dot{w}_{1} \dot{w}_{2} \ddot{w}_{2}\right)\right]+\frac{D w_{1}}{R_{\phi}^{4} E t}=0$

$R_{\phi}^{2}\left[\frac{1}{6}\left(3-2 \Delta q_{t}^{2}\right) \dddot{w}_{0}+\frac{1}{24}\left(12-7 \Delta q_{t}^{2}\right) \dddot{w}_{2}\right]-\left[\frac{9}{8} \dot{w}_{2}^{2} \ddot{w}_{2}-\frac{q_{t} R_{\phi}}{3 L}\left(3-\Delta q_{t}^{2}\right) \ddot{w}_{1}\right.$

$+\frac{3}{4}\left(\dot{w}_{1}^{2} \ddot{w}_{0}+2 \dot{w}_{0} \dot{w}_{1} \ddot{w}_{1}+2 \dot{w}_{0}^{2} \ddot{w}_{2}+4 \dot{w}_{0} \dot{w}_{2} \ddot{w}_{0}\right)+\frac{1}{2}\left(\dot{u} \ddot{w}_{1}+\ddot{u} \dot{w}_{1}\right)+$

$\left.\frac{3}{4}\left(\ddot{w}_{2} \dot{w}_{1}^{2}+2 \dot{w}_{1} \ddot{w}_{1} \dot{w}_{2}\right)\right]+\frac{16 D w_{2}}{R_{\phi}^{4} E t}=0$

$\ddot{u}+\frac{1}{2}\left[\ddot{w}_{1}\left(2 \dot{w}_{0}+\dot{w}_{2}\right)+\dot{w}_{1}\left(2 \ddot{w}_{0}+\ddot{w}_{2}\right)\right]-$

$\left[\frac{2 G}{E R_{\phi}}+\frac{2 P}{E t}\left(1-\frac{1}{9} \Delta^{2} q_{t}^{4}\right)\right]\left[\frac{u}{R_{\phi}}+\dot{w}_{0}+\left(q_{s}-q_{t}\right)\left(\frac{2 z}{L}-1\right)\right]=0$

Additionally, we minimize $\Pi$ with respect to the two parameters $q_{s}$ and $q_{t}$ and these requirements, that is, $\frac{\partial \Pi}{\partial q_{s}}=0$ and $\frac{\partial \Pi}{\partial q_{t}}=0$, give rise to two integral constraints.

$$
\int_{0}^{L}\left\{\left[\frac{2 G}{E R_{\phi}}+\frac{2 P}{E t}\left(1-\frac{1}{9} \Delta^{2} q_{t}^{4}\right)\right]\left(1-\frac{2 z}{L}\right)\left[\left(q_{s}-q_{t}\right)\left(1-\frac{2 z}{L}\right)-\left(\frac{u}{r}+\dot{w}_{0}\right)\right]+\frac{2 R_{\phi}^{2}}{L^{2}} q_{s}\left(2-\Delta q_{t}^{2}\right)\right\} \mathrm{d} z=0
$$




$$
\begin{aligned}
& \int_{0}^{L}\left\{-R_{\phi}^{2} \Delta q_{t}\left(\frac{2 q_{s}^{2}}{L^{2}}+\frac{1}{2} \ddot{w}_{0}^{2}+\frac{5}{12} \ddot{w}_{1}^{2}+\frac{2}{3} \ddot{w}_{0} \ddot{w}_{2}+\frac{7}{24} \ddot{w}_{2}^{2}\right)+\frac{4 R_{\phi}^{2}}{L^{2}} q_{t}\left(1-\Delta q_{t}^{2}\right)-\frac{2 R_{\phi}}{L} \dot{w}_{0} \dot{w}_{1}+\right. \\
& \frac{3 R_{\phi} q_{t}^{2} \Delta}{2 L} \dot{w}_{0} \dot{w}_{1}-\frac{R_{\phi}}{L} \dot{w}_{1} \dot{w}_{2}+\frac{R_{\phi} q_{t}^{2} \Delta}{L} \dot{w}_{1} \dot{w}_{2}+\left[\frac{2 G}{E}+\frac{2 P R_{\phi}}{E t}\left(1-\frac{1}{9} \Delta^{2} q_{t}^{4}\right)\right]\left(1-\frac{2 z}{L}\right)\left[\left(\frac{u}{r}+\dot{w}_{0}\right)\right. \\
& \left.-\left(1-\frac{2 z}{L}\right)\left(q_{s}-q_{t}\right)\right]-\frac{8 P R_{\phi}}{9 E t} \Delta^{2} q_{t}^{3}\left[2\left(q_{s}-q_{t}\right)^{2}+\frac{2 u^{2}}{R_{\phi}^{2}}+2 \dot{w}_{0}^{2}+\dot{w}_{1}^{2}+\dot{w}_{2}^{2}+\frac{8 \dot{w}_{0} z}{L}\left(q_{s}-q_{t}\right)+\right. \\
& \left.\frac{8 z^{2}}{L^{2}}\left(q_{s}-q_{t}\right)^{2}+\frac{4 u}{R_{\phi}}\left[\dot{w}_{0}+\frac{2 z}{L}\left(q_{s}-q_{t}\right)\right]-4\left(q_{s}-q_{t}\right)\left[\frac{u}{R_{\phi}}+\dot{w}_{0}+\frac{2 z}{L}\left(q_{s}-q_{t}\right)\right]\right]+\frac{2 P R_{\phi}^{2}}{E t L}(1 \\
& \left.\left.-\frac{1}{9} \Delta^{2} q_{t}^{4}\right)-\frac{2 M}{\pi R_{\phi} E t L}-\frac{8 P R_{\phi}}{9 E t} \Delta^{2} q_{t}^{3}\left(\frac{\dot{u}}{r}+\frac{2 q_{t}}{L}\right)\right\} \mathrm{d} z=0
\end{aligned}
$$

\section{References}

[1] Iijima, S. Helical microtubules of graphitic carbon. Nature 1991; 354: 56-58.

[2] Tombler, T., Zhou, C., Alexseyev, L., et al. Reversible electromechanical characteristics of carbon nanotubes under local-probe manipulation. Nature 2000; 405: 769-772.

[3] Liu, J. Z., Zheng, Q., Jiang, Q. Effect of a rippling mode on resonances of carbon nanotubes. Phys. Rev. Lett. 2001; 86(21): 4843.

[4] Wadee M. K., Wadee M. A., Bassom A. P., Aigner A. A. Longitudinally inhomogeneous deformation patterns in isotropic tubes under pure bending. Proc. R. Soc. London A 2006; 462: 817-838.

[5] Wang C. G, Liu Y. P., Lan L., Tan H. F. Graphene wrinkling: formation, evolution and collapse. Nanoscale 2013; 5(10): 4454-4461.

[6] Wang C. G, Lan L., Liu Y. P., Tan H. F., He X. D. Vibration characteristics of wrinkled single-layered graphene sheets. Int. J. Solid Struct. 2013; 50(10): 1812-1823.

[7] Weick, G., von Oppen, F., Pistolesi, F. Euler buckling instability and enhanced current blockade in suspended single-electron transistors. Phys. Rev. B 2011; 83: 035420.

[8] Wang, Q. Transportation of hydrogen molecules using carbon nanotube in torsion. Carbon 2009; 47: 1870-1873.

[9] Buehler M. J., Kong J., Gao H. J. Deformation mechanism of very long single-wall carbon nanotubes subject to compressive loading. J. Eng. Mater. Technol. 2004; 126: 245-249.

[10] Wang, Q. Torsional buckling of double-walled carbon nanotubes. Carbon 2008; 46: 1172-1174.

[11] Batra R. C., Sears A. Continuum models of multi-walled carbon nanotubes. Int. J. Solid Struct. 2007; 44(22): 7577-7596.

[12] Kutana A., Giapis K. P. Transient deformation regime in bending of single-walled carbon nanotubes. Phys. Rev. Lett. 2006; 97: 245501.

[13] Nikiforov I., Zhang D. B, James R. D, Dumitrică T. Wavelike rippling in multiwalled carbon nanotubes under pure bending. Appl. Phys. Lett. 2010; 96(12): 123107.

[14] Arroyo M., Belytschko T. Nonlinear mechanical response and rippling of thick multiwalled carbon nanotubes. Phys. Rev. Lett. 2003; 91: 215505.

[15] Shima, H. Buckling of carbon nanotubes: a state of the art review. Materials 2012; 5: 47-84.

[16] Poncharal P., Wang Z., Ugarte D., de Heer W. Electrostatic deflections and electromechanical resonances of carbon nanotubes. Science 1999; 283: 1513-1516.

[17] Yap H. W., Lakes R. S., Carpick R. W. Mechanical instabilities of individual multiwalled carbon nanotubes under cyclic axial compression. Nano Lett. 2007; 7: 1149-1154.

[18] Wang C. M., Zhang Y. Y., Xiang Y., Reddy J. N. Recent studies on buckling of carbon nanotubes. Appl. Mech. Rev. 2010; 63(3): 030804.

[19] $\mathrm{Ru}$ C. Q. Column buckling of multiwalled carbon nanotubes with interlayer radial displacements. Phys. Rev. B 2000; 62(24): 16962. 
[20] Wang C. M., Zhang Y. Y., He X. Q. Vibration of nonlocal Timoshenko beams. Nanotechnology 2007; 18(10): 105401.

[21] Shen H. S. Postbuckling prediction of double-walled carbon nanotubes under hydrostatic pressure. Int. J. Solid Struct. 2004; 41(9): 2643-2657.

[22] He X. Q., Kitipornchai S., Liew K. M. Buckling analysis of multi-walled carbon nanotubes: a continuum model accounting for van der Waals interaction. J. Mech. Phys. Solids 2005; 53(2): 303-326.

[23] Zhang Y. Y., Wang C. M., Challamel N. Bending, buckling, and vibration of micro/nanobeams by hybrid nonlocal beam model. J. Eng. Mech. 2009; 136(5): 562-574.

[24] Shen H. S., Zhang C. L. Torsional buckling and postbuckling of double-walled carbon nanotubes by nonlocal shear deformable shell model. Compos. Struct. 2010; 92(5): 1073-1084.

[25] Simsek M., Yurtcu H. H. Analytical solutions for bending and buckling of functionally graded nanobeams based on the nonlocal Timoshenko beam theory. Compos. Struct. 2013; 97: 378-386.

[26] Li C., Chou T. W. A structural mechanics approach for the analysis of carbon nanotubes. Int. J. Solid Struct. 2003; 40(10): 2487-2499.

[27] Ostanin I., Ballarini R., Potyondy D., Dumitrică T. A distinct element method for large scale simulations of carbon nanotube assemblies. J. Mech. Phys. Solids 2013; 61(3): 762-782.

[28] Brazier L. G. On the flexure of thin cylindrical shells and other" thin" sections. Proc. R. Soc. Ser. A 1927; 104-114.

[29] Reissner E. On finite pure bending of cylindrical tubes. Osterr. Ing. Arch. 1961; 15: 165-172.

[30] Aksel'rad E. L. Flexure and instability of thin-walled pressurized tubes. Izvestiya Akademii Nauk SSSR, Otdelenie Tekhnicheskikh Nauk, Mekhanika i Mashinostroenie 1962, 1: 98-114.

[31] Karamanos S. A, Tassoulas J. L. Stability of inelastic tubes under external pressure and bending. J. Eng. Mech. ASCE 1991, 117(12): 2845-2861.

[32] Houliara S., Karamanos S. A. Buckling and post-buckling of long pressurised elastic thin-walled tubes under in-plane bending. Int J Nonlinear Mech. 2006, 41: 491-511.

[33] Houliara S., Karamanos S. A. Stability of long transversely-isotropic elastic cylindrical shells under bending. Int J Solids Struct. 2010, 47:10-24.

[34] Pantano A., Boyce M. C., Parks D. M. Nonlinear structural mechanics based modeling of carbon nanotube deformation. Phys. Rev. Lett. 2003; 91: 145501-145504.

[35] Li X. Y., Yang W., Liu B. Bending induced rippling and twisting of multiwalled carbon nanotubes. Phys. Rev. Lett. 2007; 98: 205502.

[36] Volkov A. N., Shiga T., Nicholson D., Shiomi J., Zhigilei L. V. Effect of bending buckling of carbon nanotubes on thermal conductivity of carbon nanotube materials. J. Appl. Phys. 2012; 111(5): 053501.

[37] Wadee M. K., Wadee M. A., Bassom A. P. Effects of orthotropy and variation of Poisson's ratio on the behaviour of tubes in pure flexure. J. Mech. Phys. Solids 2007; 55(5): 1086-1102.

[38] Le van A., Wielgosz C. Bending and buckling of inflatable beams: some new theoretical results. Thin-Walled Struct. 2005; 43(8): 1166-1187.

[39] Doedel E. J., Oldeman B. E. Auto-07P: Continuation and bifurcation software for ordinary differential equations. Tech. Rep.; Department of Computer Science, Concordia University, Montreal, Canada; 2009.

[40] Silvestre N. Length dependence of critical measures in single-walled carbon nanotubes. Int. J. Solid Struct. 2008; 45(18): 4902-4920.

[41] Cohen A. E., Mahadevan L. Kinks, rings, and rackets in filamentous structures. Proc. Nat. Acad. Sci. 2003; 100(21): 12141-12146.

[42] Giusca C. E., Tison Y., Silva S. R. P. Evidence for metal-semiconductor transitions in twisted and collapsed double-walled carbon nanotubes by scanning tunneling microscopy. Nano Lett. 2008; 8: 3350-3356.

[43] Diniz E. M., Nunes R. W., Chacham H., Mazzoni M. S. C. Bistability, softening, and quenching of magnetic moments in Ni-filled carbon nanotubes. Phys. Rev. B 2010; 81: 153413.

[44] Huang X., Zhang S. Load-driven morphological evolution in covalently bridged multiwalled carbon nanotubes. Appl. Phys. Lett. 2010; 96: 203106. 
[45] Arroyo M., Arias I. Rippling and a phase-transforming mesoscopic model for multiwalled carbon nanotubes. J. Mech. Phys. Solids 2008; 56(4): 1224-1244.

[46] Abeyaratne R., Knowles J. Evolution of Phase Transitions: A Continuum Theory. Cambridge University Press, Cambridge; 2006.

[47] Dumitrică T., James R. D. Objective molecular dynamics. J. Mech. Phys. Solids 2007; 55(10): 2206-2236.

[48] Cao G., Chen X. Buckling of single-walled carbon nanotubes upon bending: Molecular dynamics simulations and finite element method. Phys. Rev. B 2006; 73(15): 155435.

[49] Chen Y. L., Liu B., Wu J., Huang Y., Jiang H., Hwang K. C. Mechanics of hydrogen storage in carbon nanotubes. J. Mech. Phys. Solids 2008; 56(11): 3224-3241.

[50] Jiang L.Y., Huang, Y., Jiang, H., Ravichandran, G., Gao, H., Hwang, K.C., Liu, B. A cohesive law for carbon nanotube/polymer interfaces based on the van der Waals force. J. Mech. Phys. Solids 2006; 54, 2436-2452.

[51] Fichter WB., 1966. A theory for inflated thin-wall cylindrical beams. NASA Technical note TN D-3466, 1966.

[52] Tersoff J. Empirical interatomic potential for carbon, with applications to amorphous carbon. Phys. Rev. Lett. 1998; 61(25) 2879.

[53] Brenner D. W. Empirical potential for hydrocarbons for use in simulating the chemical vapor deposition of diamond films. Phys. Rev. B 1990; 42(15): 9458.

[54] Huang Y., Wu J., Hwang K. C. Thickness of graphene and single-wall carbon nanotubes. Phys. Rev. B 2006; 74(24): 245413.

[55] Davids W. G., Zhang H. Beam finite element for nonlinear analysis of pressurized fabric beam-columns. Eng. Struct. 2008; 30(7): 1969-1980. 